
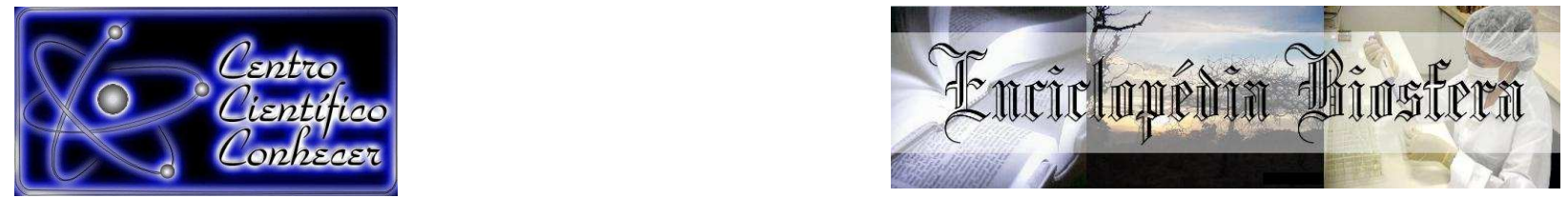

\title{
INFLUÊNCIA DO MANEJO DE PLANTAS DANINHAS NA INCIDÊNCIA DO BICHO-MINEIRO LEUCOPTERA COFFEELLA (GUÉRIN-MÈNEVILLE, 1842) (LEPIDOPTERA: LYONETIIDAE) NA REGIÃO DO CERRADO MINEIRO
}

\footnotetext{
Stéfane Carolina Quista da Silva Faria ${ }^{1}$, Marina Robles Angelini ${ }^{2}$

1- Discente do curso de Engenharia Agronômica do Instituto Federal do Triângulo Mineiro - Campus Uberlândia (stefanecfaria@hotmail.com) Uberlândia - Brasil

2- Professora Doutora do Instituto Federal do Triângulo Mineiro (IFTM), Campus Uberlândia
}

\section{Recebido em: 08/09/2015 - Aprovado em: 14/11/2015 - Publicado em: 01/12/2015 DOI: http://dx.doi.org/10.18677/Enciclopedia_Biosfera_2015_180}

\begin{abstract}
RESUMO
A cafeicultura apresenta problemas fitossanitários que causam queda da produtividade, bem como interferem na qualidade da bebida. Em cafezais, a influência do mato na regulação populacional de artrópodes-praga é importante ferramenta do manejo, pela atração e manutenção dos organismos benéficos na cultura, aumentando o controle biológico natural. Um dos principais problemas fitossanitários da cultura é o ataque de pragas, principalmente do bicho-mineiro-docafeeiro, Leucoptera coffeella (Guérin-Mèneville, 1842) (Lepidoptera: Lyonetiidae). $O$ presente estudo avaliou os efeitos de diferentes manejos de plantas daninhas na incidência do bicho-mineiro na região do Cerrado mineiro. Os tratamentos constaram de diferentes manejos de plantas daninha: T1- sem capina, T2 - roçada da entrelinha com triturador 1400 mm com varredor (TM 5.1 - 1400 Dragão Sol®), T3 controle químico das plantas daninhas na entrelinha, T4 - roçada convencional da entrelinha (TM 5.1 - 1400 Dragão Sol® sem o acionamento do varredor). A ausência de plantas infestantes na entrelinha do cafeeiro favoreceu o aumento populacional de $L$. coffeella, sendo observada uma maior incidência quando utilizado o controle químico para eliminação das plantas infestantes.
\end{abstract}

PALAVRAS-CHAVE: Manejo Integrado de pragas, Leucoptera coffeella, plantas infestantes.

\section{PLANT MANAGEMENT INFLUENCE OF WEEDS IN EFFECT OF COFFEE LEAF- MINER LEUCOPTERA COFFEELLA (LEPIDOPTERA: LYONETIIDAE) IN CERRADO MINEIRO}

\begin{abstract}
The coffee has phytosanitary problems that cause drop in productivity as well as interfere with the quality of the beverage. In coffee plantations, the influence of the bush in population control of arthropod pests is important management tool for the attraction and maintenance of beneficial organisms in culture, increasing the natural biological control. One of the main phytosanitary problems of culture is the attack of pests, especially the coffee leaf-miner, Leucoptera coffeella (Guérin-Mèneville, 1842) (Lepidoptera: Lyonetiidae). This study evaluated the effects of different weed
\end{abstract}


management systems in the incidence of leaf-miner in cerrado mineiro. The treatments consisted of different managements of weed plants: no weeding T1, T2 cutting weeds with the brush shredder $900 \mathrm{~mm}$ with driven brush and turbine (TM 5.1 - 900 Dragon Sol®), T3 - chemical control of weeds between rows, T4 - Conventional mowing the leading (TM 5.1 - 900 Dragon Sol® without driven brush and turbine). The absence of weeds in the coffee leading favored the population growth of $L$. coffeella, being observed a higher incidence when used chemical control for removal of weeds.

KEYWORDS: Integrated pest management, Leucoptera coffeella, weeds.

\section{INTRODUÇÃO}

A cafeicultura é uma atividade econômica de grande destaque na economia mundial que contribuiu decisivamente para o desempenho da economia dos maiores produtores: Brasil, Vietnã e Colômbia (USDA, 2015). Devido ao grande valor cultural, social e econômico do café, devem ser adotados novos procedimentos e tecnologias para que se possa reduzir as perdas e os custos de produção (CUNHA et al., 2011).

Essa cultura apresenta problemas fitossanitários que causam queda da produtividade, bem como interferem na qualidade da bebida. Um dos principais problemas é o ataque de pragas. O bicho-mineiro, Leucoptera coffeella (GuérinMéneville, 1842) (Lepidoptera: Lyonetiidae) é considerada a principal praga da cultura no Brasil (REIS et al., 2010). As fêmeas desse inseto ovipositam na superfície superior das folhas e após a eclosão, as lagartas penetram na epiderme da folha ocasionando lesões pela alimentação das lagartas, causando desfolha e redução da área fotossintética. Os sintomas são mais visíveis no terço superior da planta onde ocorre grande desfolha em altas infestações (SOUZA \& REIS, 2000).

Atualmente são conhecidos diversos métodos para o controle do bichomineiro, como o químico, o biológico por ação de predadores e parasitoides e o cultural. Para que haja uma melhoria no processo produtivo, há uma necessidade de adoção de estratégias para o manejo das pragas que atacam a cultura do café, onde se destaca o manejo cultural, que talvez possa contribuir para a manutenção da população desse inseto abaixo do nível de dano econômico (REIS et al., 2002).

O controle cultural, que objetiva a manutenção das condições ambientais, auxilia na redução do ataque da praga à cultura e também oferece condições para o abrigo e alimentação de inimigos naturais (REIS et al., 2002). Assim deve ser dada especial atenção às plantas que vegetam na rua do cafeeiro, para manutenção e aumento de inimigos naturais das pragas do café, principalmente do bicho-mineiro colaborando com a realização do controle biológico e diminuindo a aplicação de defensivos agrícolas.

O controle dos insetos-praga que atingem o cafeeiro deve ser estabelecido de maneira integrada, pela associação entre os diversos métodos de controle já existentes. Sendo assim, o presente trabalho foi desenvolvido a fim de se avaliar os efeitos da presença e do manejo de plantas infestantes na incidência do bicho mineiro na região do Cerrado mineiro.

\section{MATERIAL E MÉTODOS}

O experimento foi conduzido na Fazenda Paraná, localizada no município de Nova Ponte, MG, situada a S 1902'29,7", W 047²6'3 2,2"e 950 m de altitude, no período de novembro de 2011 a julho de 2012, utilizando a cultivar Mundo Novo 
plantada com espaçamento de $4 \mathrm{~m}$ entre linhas e $1 \mathrm{~m}$ entre plantas, totalizando 2500 plantas.ha' ${ }^{-1}$.

O delineamento experimental foi o de blocos ao acaso com 4 tratamentos e 5 repetições, totalizando 20 parcelas, onde cada uma delas foi constituída de três linhas de plantio e 50 metros de comprimento, sendo a área útil a ser considerada para avaliação de 30 metros. Avaliou-se a linha de café central da parcela.

Para avaliar a influência de tratos culturais na incidência de $L$. coffeella, os tratamentos constaram de diferentes manejos de plantas daninha: T1- sem capina, T2 - roçada da entrelinha com triturador $1400 \mathrm{~mm}$ com varredor (TM 5.1- 1400 Dragão SolB), T3 - controle químico das plantas daninhas na entrelinha (Glifosato), T4 - roçada convencional (Triturador TM 5.1- 1400 Dragão Solß sem o acionamento do varredor). $\mathrm{Na}$ avaliação da incidência da praga foram efetuadas, mensalmente, coletas de amostras de folhas, retirando-se ao acaso 100 folhas por parcela, na região mediana-superior dos ramos (terceiro ou quarto par de folhas completamente desenvolvidas). Após a coleta, as folhas foram acondicionadas em sacos de papel, perfurados e codificados com o tratamento correspondente.

As variáveis avaliadas foram: número de folhas com minas (folhas minadas), número de minas, número de folhas com minas vivas e número de larvas vivas. Para a contagem do número de folhas com minas foram considerados todos os tipos de minas, ou seja, intactas, abertas, velhas, parasitadas e com larva viva. Para contagem das minas com larva viva, as minas foram abertas com auxílio de estilete ou agulha, tomando-se o máximo de cuidado para não danificar as possíveis larvas presentes.

\section{RESULTADOS E DISCUSSÃO}

Através dos resultados obtidos observa-se na $1^{\underline{a}}$ e $2^{\underline{a}}$ avaliações realizadas que a porcentagem de folhas minadas não diferiu estatisticamente entre os

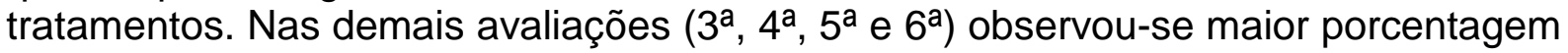
de folhas com minas quando se utilizou o controle químico, mostrando que a ausência de plantas daninhas na área favorece o aumento populacional de $L$. coffeella (Tabela 1). Esse fato pode estar relacionado à diminuição de inimigos naturais quando a cultura é mantida no limpo. Estudos realizados por FADINI et al. (2001) mostram que o aumento da diversidade das espécies vegetais pode resultar na diminuição de algumas pragas, devido ao aumento da diversidade e abundância de artrópodes considerados inimigos naturais de pragas agrícolas.

TABELA 1. Porcentagem média de folhas de cafeeiro com minas de Leucoptera coffeella. Nova Ponte/MG, 2012.

\begin{tabular}{|c|c|c|c|c|c|c|}
\hline Tratamento & $\begin{array}{l}1^{\underline{a}} \\
\text { avaliação }\end{array}$ & $\begin{array}{l}2^{\mathrm{a}} \\
\text { avaliação }\end{array}$ & $\begin{array}{l}3^{\mathbf{a}} \\
\text { avaliação }\end{array}$ & $\begin{array}{l}4^{\mathrm{a}} \\
\text { avaliação }\end{array}$ & $\begin{array}{l}5^{\mathrm{a}} \\
\text { avaliação }\end{array}$ & $\begin{array}{l}6^{\mathrm{a}} \\
\text { avaliação }\end{array}$ \\
\hline Sem capina & $0,4 a$ & $4,8 a$ & $5,2 b$ & $6,4 \mathrm{~b}$ & $2,0 c$ & $4,4 b$ \\
\hline $\begin{array}{l}\text { Roçada da entrelinha com } \\
\text { triturador TM } 5.1-1400 \\
\text { com varredor }\end{array}$ & $1,6 a$ & $3,2 \mathrm{a}$ & $4,0 \mathrm{~b}$ & $6,8 b$ & $2,4 \mathrm{c}$ & $4,4 b$ \\
\hline $\begin{array}{l}\text { Controle químico na } \\
\text { entrelinha (glifosato) }\end{array}$ & $0,8 a$ & $2,8 a$ & $8,0 a$ & $12,8 a$ & $6,4 a$ & $10,8 a$ \\
\hline $\begin{array}{l}\text { Roçada convencional da } \\
\text { entrelinha com triturador } \\
\text { TM } 5.1-1400 \text { sem } \\
\text { varredor }\end{array}$ & $0,4 a$ & $2,4 a$ & $6,4 a$ & $8,8 b$ & $3,8 b$ & $5,6 b$ \\
\hline CV $(\%)$ & 31,98 & 28,35 & 13,52 & 11,42 & 20,01 & 29,41 \\
\hline
\end{tabular}


Em relação ao número de minas, não houve diferença significativa entre os tratamentos na $1^{\underline{a}}$ e $2^{a}$ avaliação. Já na $3^{\underline{a}}$ avaliação, nota-se um maior número de minas nos tratamentos T1 (testemunha) e T2 (roçada da entrelinha com triturador e varredor), os quais diferiram significativamente dos tratamentos T3 (controle químico das plantas daninhas) e T4 (roçada convencional). Na $4^{a}$ avaliação, o Tratamento T1 (testemunha) apresentou maior número de folhas com minas, diferindo estatisticamente dos demais. $\mathrm{Na} 5^{\underline{a}}$ e $6^{\underline{a}}$ avaliação o maior número de minas foi observado no tratamento T3 (controle químico de plantas daninhas) (Tabela 2). No tratamento onde foram mantidas as plantas daninhas, observa-se que esse número foi significativamente menor, fato também relatado por ANDOW (1991) que concluiu que os herbívoros monófagos tendem a ter menor população em sistemas diversificados. Entretanto, estudos realizados por AMARAL et al. (2010) mostraram que a infestação do bicho-mineiro não foi influenciada pelo aumento da diversidade de plantas associadas ao cafeeiro.

TABELA 2. Número de minas de Leucoptera coffeella em folhas de cafeeiro. Nova Ponte/MG,

\begin{tabular}{|c|c|c|c|c|c|c|}
\hline Tratamento & $\begin{array}{l}1 \underline{\underline{a}} \\
\text { avaliação }\end{array}$ & $\begin{array}{l}2^{\mathbf{a}} \\
\text { avaliação }\end{array}$ & $\begin{array}{l}3^{\underline{a}} \\
\text { avaliação }\end{array}$ & $\begin{array}{l}4^{\underline{a}} \\
\text { avaliação }\end{array}$ & $\begin{array}{l}5^{\mathbf{a}} \\
\text { avaliação }\end{array}$ & $\begin{array}{l}6^{\mathbf{a}} \\
\text { avaliação }\end{array}$ \\
\hline Sem capina & $0,8 \mathrm{a}$ & $2,6 a$ & $4,2 \mathrm{a}$ & $6,8 a$ & $1,8 \mathrm{c}$ & $2,4 \mathrm{~b}$ \\
\hline $\begin{array}{l}\text { Roçada da entrelinha com } \\
\text { triturador TM } 5.1-1400 \\
\text { com varredor }\end{array}$ & $0,4 a$ & $1,8 a$ & $3,8 a$ & $4,8 b$ & $2,8 c$ & $2,4 b$ \\
\hline $\begin{array}{l}\text { Controle químico na } \\
\text { entrelinha (glifosato) }\end{array}$ & $0,2 a$ & $1,4 a$ & $2,8 b$ & $4,2 b$ & $6,8 a$ & $5,6 a$ \\
\hline $\begin{array}{l}\text { Roçada convencional da } \\
\text { entrelinha com triturador } \\
\text { TM } 5.1-1400 \text { sem } \\
\text { varredor }\end{array}$ & $0,2 a$ & $1,2 \mathrm{a}$ & $2,2 \mathrm{~b}$ & $3,4 b$ & $4,0 \mathrm{~b}$ & $3,6 b$ \\
\hline $\mathrm{CV}(\%)$ & 22,3 & 27,34 & 14,62 & 28,78 & 20,67 & 29,51 \\
\hline
\end{tabular}

Médias seguidas da mesma letra na coluna não diferem entre si pelo teste de Scott-Knott a 5\% de probabilidade.

Quanto à porcentagem de folhas com larvas vivas de L. coffeela, não se observou diferença significativa entre os tratamentos na $1^{\underline{a}}$, $2^{\underline{a}}$ e $3^{\underline{a}}$ avaliação. Nas demais avaliações com o uso de tratamento químico para o controle das plantas daninhas, a porcentagem de folhas com larvas vivas foi estatisticamente maior em relação aos demais tratamentos. $\mathrm{Na} 4^{\underline{a}}$ avaliação não houve diferença entre 0 tratamento com controle químico (T3) e o tratamento onde se fez a roçada convencional (Tabela 3).

TABELA 3. Porcentagem de folhas de cafeeiro com larvas vivas de Leucoptera coffeella. Nova Ponte/MG, 2012.

\begin{tabular}{|c|c|c|c|c|c|c|}
\hline Tratamento & $\begin{array}{c}1^{\underline{a}} \\
\text { avaliação }\end{array}$ & $\begin{array}{c}2^{\underline{a}} \\
\text { avaliação }\end{array}$ & $\begin{array}{c}3^{\underline{a}} \\
\text { avaliação }\end{array}$ & $\begin{array}{c}4^{\underline{a}} \\
\text { avaliação }\end{array}$ & $\begin{array}{c}5^{\mathbf{a}} \\
\text { avaliação }\end{array}$ & $\begin{array}{c}6^{\underline{\mathbf{a}}} \\
\text { avaliação }\end{array}$ \\
\hline Sem capina & $0,0 \mathrm{a}$ & $4,0 \mathrm{a}$ & $5,3 a$ & $2,4 \mathrm{~b}$ & $1,2 \mathrm{c}$ & $1,2 d$ \\
\hline $\begin{array}{l}\text { Roçada da entrelinha com } \\
\text { triturador TM } 5.1 \text { - } 1400 \text { com } \\
\text { varredor }\end{array}$ & $0,4 a$ & $2,0 a$ & $4,0 \mathrm{a}$ & $3,2 b$ & $3,6 b$ & $3,2 \mathrm{c}$ \\
\hline $\begin{array}{l}\text { Controle químico na entrelinha } \\
\text { (glifosato) }\end{array}$ & $0,0 \mathrm{a}$ & $2,8 a$ & $4,0 \mathrm{a}$ & $6,8 a$ & $9,2 a$ & $7,2 a$ \\
\hline $\begin{array}{l}\text { Roçada convencional da } \\
\text { entrelinha com triturador TM } \\
5.1-1400 \text { sem varredor }\end{array}$ & $0,0 \mathrm{a}$ & $3,2 a$ & $7,3 a$ & $5,6 a$ & $4,8 b$ & $5,2 b$ \\
\hline $\mathrm{CV}(\%)$ & 26,03 & 37,92 & 22,23 & 18,38 & 21,35 & 29,16 \\
\hline
\end{tabular}

Médias seguidas da mesma letra na coluna não diferem entre si pelo teste de Scott-Knott a 5\% de probabilidade. 
Outro parâmetro avaliado foi o número de larvas vivas em cada tratamento. $\mathrm{Na} 1^{\mathrm{a}}$ e $2^{\underline{a}}$ avaliação os tratamentos não apresentaram influência desse parâmetro. $\mathrm{Na} 3^{\mathrm{a}}$ e $4^{\mathrm{a}}$ avaliação podemos observar maior número de larvas vivas no Tratamento T3, no qual se utilizou o controle químico das plantas daninhas, no entanto, não houve diferença desse tratamento com aquele em que foi realizada a roçada convencional (T4). Na $5^{\text {a }}$ avaliação maior número de larvas vivas também foi observado no Tratamento T3, o qual diferiu significativamente dos demais. Na $6^{\mathbf{a}}$ avaliação resultados semelhantes foram encontrados, no entanto, não houve diferença entre os Tratamentos T3 e T4 (Tabela 4). Através desse parâmetro podemos observar que a partir da 3a avaliação a presença de plantas daninhas na entre linha do cafezal levou a diminuição do número de larvas vivas de $L$. coffeella. Manejo este recomendado por MATIELLO et al. (2010) que destacam o uso racional de capinas, cobertura morta e culturas intercalares para que sejam preservados possíveis parasitas e predadores do bicho-mineiro.

TABELA 4. Número médio de larvas vivas de Leucoptera coffeella. Nova Ponte/MG, 2012.

\begin{tabular}{|c|c|c|c|c|c|c|}
\hline Tratamento & $\begin{array}{l}1^{\underline{a}} \\
\text { avaliação }\end{array}$ & $\begin{array}{l}2^{\underline{a}} \\
\text { avaliação }\end{array}$ & $\begin{array}{l}3^{\underline{a}} \\
\text { avaliação }\end{array}$ & $\begin{array}{l}4^{\underline{a}} \\
\text { avaliação }\end{array}$ & $\begin{array}{l}5^{\underline{a}} \\
\text { avaliação }\end{array}$ & $\begin{array}{l}6^{\mathbf{a}} \\
\text { avaliação }\end{array}$ \\
\hline Sem capina & $0,0 \mathrm{a}$ & $2,4 a$ & $2,0 b$ & $1,2 \mathrm{~b}$ & $0,6 \mathrm{c}$ & $0,6 \mathrm{c}$ \\
\hline $\begin{array}{l}\text { Roçada da entrelinha com } \\
\text { triturador TM } 5.1-1400 \\
\text { com varredor }\end{array}$ & $0,2 a$ & $1,0 a$ & $2,2 b$ & $1,8 b$ & $1,8 b$ & $1,6 b$ \\
\hline $\begin{array}{l}\text { Controle químico na } \\
\text { entrelinha (glifosato) }\end{array}$ & $0,0 \mathrm{a}$ & $1,8 a$ & $4,4 a$ & $3,8 \mathrm{a}$ & $4,6 a$ & $3,8 a$ \\
\hline $\begin{array}{l}\text { Roçada convencional da } \\
\text { entrelinha com triturador } \\
\text { TM } 5.1-1400 \text { sem } \\
\text { varredor }\end{array}$ & $0,0 \mathrm{a}$ & $1,4 a$ & $3,2 a$ & $3,2 \mathrm{a}$ & $3,2 a$ & $3,2 a$ \\
\hline $\mathrm{CV}(\%)$ & 15,79 & 37,02 & 15,86 & 19,3 & 17,9 & 16,46 \\
\hline
\end{tabular}

\section{CONCLUSÕES}

A ausência de plantas infestantes na entrelinha do cafeeiro favoreceu 0 aumento populacional de $L$. coffeella, sendo observada uma maior incidência quando utilizado o controle químico para eliminação das plantas infestantes.

\section{REFERÊNCIAS}

AMARAL, D. S.; VENZON, M.; PALLINI, A.; LIMA, P. C.; DESOUZA, O. A diversificação da vegetação reduz 0 ataque do bicho-mineiro-do-cafeeiro. Leucoptera coffeella (Guérin-mèneville) (Lepidoptera: Lyonetiidae). Neotropical Entomolology, v. 39, n. 4, p. 543-548, 2010.

ANDOW, D. A. Vegetacional diversity and arthropod population response. Annual Review of Entomology, v. 36, p. 561-586, 1991.

CUNHA, J. P. A. R. da; GITIRANA NETO, J.; BUENO, M. R. Evaluation of a device for the application of pesticides on mechanized coffee crops (Coffea arabica L.). Interciencia, v.36, p.312-316, 2011. 
FADINI, M. A.; REGINA, M. A.; FRÁGUAS, J.C.; LOUZADA, J.N.C. Efeito da cobertura vegetal do solo sobre a abundância e diversidade de inimigos naturais de pragas em vinhedos. Revista brasileira de fruticultura, Jaboticabal, v. 23, n. 3, p. 575-576, 2001.

MATIELLO, J. B.; SANTINATO, R.; GARCIA, A. W. R; ALMEIDA, S. R.; FERNANDES, D. R. Cultura de Café no Brasil: Manual de Recomendações. Ed. 2010. MAPA/PROCAFÉ, 2010. 542 p.

REIS, P. R.; SOUZA, J. C; SANTACECÍLIA.; L. V. C.; SILVA, A.; ZACARIAS, M. S. Manejo integrado de pragas do cafeeiro. In: REIS, P.R.; CUNHA, R.L. Café arábica: do plantio à colheita. Lavras: Epamig, p. 573-688. 2010.

REIS, P. R.; SOUZA, J. C.; VENZON, M. Manejo das principais pragas do cafeeiro. Informe Agropecuário, Belo Horizonte, v. 23, n. 214/215, p. 83-99. 2002.

SOUZA, J.C.; REIS, P.R. Pragas do cafeeiro: reconhecimento e controle. Viçosa: CTP, 2000. 154p.

UNITED STATES DEPARTMENT OF AGRICULTURE - USDA. Coffee: World Markets and Trade. Foreign Agricultural Service, jun. 2015. 